\title{
Production Planning Based on a Genetic Algorithm Controlled Simulation Model
}

\author{
Alexey N. Sochnev* \\ Siberian Federal University \\ Krasnoyarsk, Russian Federation
}

Received 04.12.2020, received in revised form 11.02.2021, accepted 21.03.2021

\begin{abstract}
The article proposes an approach to solving the task of operational calendar planning of production based on the application of the principles of the optimization and simulation approach. The production simulation model is implemented using the Tecnomatix Plant Simulation software. The optimization procedure is represented by a genetic algorithm. In the implementation of the genetic algorithm, a simulation model is used to evaluate fitness functions. An example of using the proposed approach for a typical production system is given and the positive effect of its application is confirmed. Features of use, positive and negative properties, as well as the possibility of replication to other types of simulation models are revealed.
\end{abstract}

Keywords: resource-constrained project scheduling problem (RCPSP), operational calendar planning, optimization and simulation approach, simulation model, genetic algorithm.

Citation: Sochnev A.N. Production planning based on a genetic algorithm controlled simulation model, J. Sib. Fed. Univ. Eng. \& Technol., 2021, 14(2), 233-243. DOI: 10.17516/1999-494X-0304

\section{Планирование производства}

\section{на основе управляемой генетическим алгоритмом имитационной модели}

\author{
А.Н. Сочнев \\ Сибирский федеральньй университет \\ Российская Федераџия, Красноярск
}

Аннотация. В статье предложен подход к решению задачи оперативно-календарного планирования производства на основе применения принципов оптимизационно-имитационного подхода. Имитационная модель производства реализована средствами программы Tecnomatix Plant

(C) Siberian Federal University. All rights reserved

This work is licensed under a Creative Commons Attribution-Non Commercial 4.0 International License (CC BY-NC 4.0).

* Corresponding author E-mail address: asochnev@sfu-kras.ru 
Simulation. Процедура оптимизации представлена генетическим алгоритмом. В реализации генетического алгоритма для оценки функции приспособленности используется имитационная модель. Приведен пример использования предложенного подхода для типичной производственной системы и подтвержден положительный эффект от его применения. Выявлены особенности использования, положительные и отрицательные свойства, а также возможности тиражирования на другие виды имитационных моделей.

Ключевые слова: задача планирования проекта с ограниченными ресурсами (RCPSP), оперативнокалендарное планирование, оптимизационно-имитационный подход, имитационная модель, генетический алгоритм.

Цитирование: Сочнев, А.Н. Планирование производства на основе управляемой генетическим алгоритмом имитационной модели / А. Н. Сочнев // Журн. Сиб. федер. ун-та. Техника и технологии, 2021, 14(2). С. 233-243. DOI: 10.17516/1999-494X-0304

\section{Введение}

Одной из наиболее важных и сложных задач оптимизации является задача RCPSP (Resource-Constrained Project Scheduling Problem) - построение оптимального расписания выполнения работ проекта с учетом отношений предшествования между работами и с учетом необходимых и (или) доступных ресурсов, при котором будет оптимизирована некоторая целевая функция (например, минимизация длительности выполнения проекта). Задача RCPSP является экстремально NP-трудной $[1,2]$. С точки зрения создания системы управления производственной системой описанная задача эквивалентна классической задаче оперативнокалендарного планирования (ОКП) производства [3].

Для решения задачи RCPSP (ОКП) разработаны множество точных и приближенных методов, которые обладают разной скоростью и точностью решения $[1,4,5]$. Основная практическая проблема, с которой сталкиваются в рассматриваемой задаче, - это то, что необходимо осуществить поиск оптимального решения в большом пространстве поиска на множестве допустимых решений за приемлемое время. При этом выбор и анализ допустимых решений является сложной комбинаторной задачей. Методы решения задачи планирования можно условно разделить на три основных класса: аналитические, имитационные и аналитико-имитационные. Аналитические методы основываются на аппарате математического программирования, например динамического программирования, дискретной оптимизации и т. д. Эти методы принципиально позволяют получить оптимальный план, однако на практике решение задач, учитывающих множество реальных факторов, становится очень трудоемким. Другой недостаток аналитических методов - «жесткость» модели. Структурно изменить однажды построенную аналитическую модель может только математик достаточно высокой квалификации. В условиях частых структурных изменений, характерных для гибкого производства, эксплуатация такой модели затруднительна и малоэффективна. Одним из наиболее известных и эффективных методов решения задачи планирования процесса является алгоритм границ и ветвей $[2,6]$.

Имитационные методы планирования предусматривают проведение имитационного эксперимента на моделях, структурно подобных моделируемому объекту или процессу. Структурное подобие модели и объекта моделирования существенно упрощает внесение текущих изменений в модель.

Имитационные методы строятся по схеме последовательного анализа вариантов на основе правил предпочтения. Эта схема связана с пошаговым конструированием вариантов с помо- 
щью моделирования, в процессе которого имитируется прохождение материальных потоков через производственный участок. При построении имитационной модели сравнительно просто учитываются такие трудноформализуемые в аналитических моделях ограничения, как структура транспортной сети, временный дефицит ресурсов, частичная и временная потеря работоспособности оборудования и ряд других.

Аналитико-имитационные методы строятся по схеме, включающей как аналитические, так и имитационные процедуры. Цель разработки таких схем - объединение достоинств обоих методов.

Известны два подхода к построению аналитико-имитационных процедур календарного планирования. Один подход предусматривает двухэтапное решение задачи. На первом этапе планируется обработка укрупненных партий изделий на двух или трех группах технологического оборудования. При этом из-за небольшой размерности целесообразно использовать точные или приближенные аналитические методы решения. На втором этапе строятся детальные календарные планы внутри каждой из выделенных групп оборудования с использованием имитационных методов.

Другой подход предполагает объединение аналитических и имитационных процедур в рамках одной модели. В данной статье предлагается для решения задачи использовать серию экспериментов с имитационной моделью, начальные параметры которой определяются с помощью генетического алгоритма $[4,7]$. В основе данной идеи лежат основные положения оптимизационно-имитационного подхода к синтезу структур управления сложными системами, предполагающего совместное использование в процессе синтеза управления оптимизационных и имитационных моделей, их рациональное взаимодействие [8, 9].

\section{1. Постановка задачи планирования}

Общая постановка задачи RCPSP выглядит следующим образом [1]. Дано множество работ, возобновляемых и невозобновляемых ресурсов. Заданы длительности выполнения для каждой работы. Во время выполнения работы требуется заданное количество единиц ресурса $k$. После завершения работы освобожденные ресурсы в полном объеме могут быть мгновенно назначены на другие работы. Между некоторыми парами работ заданы ограничения предшествования. Требуется определить моменты времени начала выполнения работ так, чтобы минимизировать время выполнения всего проекта. При этом необходимо выполнение ограничений, связанных с использованием ресурсов и следования работ.

Формально задача планирования процесса ставится следующим образом [3]. Пусть на участке требуется обработать $n$ деталей (или их партий). Обозначим $L_{i j}=\left(q_{i j}, t_{i j}\right)$ операцию с номером $i\left(i=\overline{1, m_{j}}\right)$, которая выполняется над $j$-й деталью $(j=\overline{1, n}) ; m_{j}$ - число операций, которое необходимо выполнить над $j$-й деталью; $q_{i j}$ - номер группы оборудования, настроенного на выполнение операции $L_{i j} ; t_{i j}$ - номинальная продолжительность выполнения операций.

Решение задачи организуется на множестве допустимых планов

$$
\Omega_{p}=\left\{P: G=\left(L_{i_{1}} ; L_{i_{2}} ; \ldots ; L_{i_{m_{j}}}\right), t_{i j}^{k}=t_{i j}^{0}+t_{i j}, t_{i_{1} j_{1}}^{0} \leq t_{i_{2} j_{2}}^{0}, i=\overline{1, m_{j}}, j=\overline{1, n},\right\},
$$

где $G$ - технологический маршрут детали; $t_{i j}^{k}$ - момент окончания операции $L_{i j} ; t_{i j}^{0}-$ момент окончания операции $L_{i j} ; t_{i_{1} j_{1}}^{0}-$ момент начала операции $i 1 ; t_{i_{2} j_{2}}^{0}-$ момент начала операции $i 2$. 
Совокупность чисел $P=\left\{t_{i j}^{0}\right\}$, удовлетворяющих ограничениям, называется календарным планом. Решение задачи календарного планирования заключается в отыскании неизвестных величин $\left\{t_{i j}^{0}\right\}$. При формальной постановке задачи календарного планирования требуется задать целевую функцию планирования, которая каждому плану $P$ ставит в соответствие некоторое число $F(P)$, оценивающее эффективность плана (критерий эффективности). Задача оптимального планирования заключается в отыскании такого плана $P^{*}$, который экстремизирует критерий эффективности $F$ и удовлетворяет ограничениям.

Критерии. Выбор критерия эффективности осуществляется индивидуально для каждого конкретного производственного подразделения по результатам анализа его экономических, организационных и технических особенностей. Наиболее часто используют в качестве критерия эффективности минимизацию времени выпуска заданного объема продукции, минимизацию максимального времени простоя, максимизацию загрузки оборудования, минимизацию суммарных производственных затрат и др. Далее в качестве критерия оптимальности выбирается минимизация общего времени производственного процесса.

Ограничения. Кроме очевидных ограничений при решении задач календарного планирования необходимо учитывать еще ряд других. Наиболее часто учитывают следующие ограничения: структура сети транспортных маршрутов, директивные сроки окончания обработки определенных деталей, многочисленные ресурсные ограничения, в том числе динамические.

\section{2. Основные положения предлагаемого подхода}

Рассматривается задача RCPSP (ОКП) в постановке, описанной выше. Исходя из представленной структуры процессов, существенной возможностью оптимизации можно признать перераспределение технологических маршрутов изделий в системе. Свободу выбора в данном случае предоставляет дублирующее друг друга технологическое оборудование (токарные станки). Также необходимо учитывать, что при определении вариантов распределения необходимо учитывать особенности технологических процессов. Например, в рассматриваемой производственной системе часть технологических процессов может выполняться только по фиксированному маршруту.

Примем для определенности, что маршруты деталей уже выбраны другим методом. В таком случае остается возможность перераспределения порядка следования изделий внутри сформированных маршрутов. Задача сводится к поиску оптимального следования изделий в группах. В общем случае маршрут может быть фиксирован только для одной партии изделий, но для упрощения решения он будет фиксирован для всех партий одного типа деталей.

Таким образом, процедура оптимизации предполагает реализацию множества повторяющихся итераций, по итогам реализации каждой из которых оценивается результат. Специфика эволюционной процедуры оптимизации в том, что требуется критерий ее остановки. В процедуре планирования производства он может быть определен экспертным методом, по условию остановки либо по количеству шагов поиска.

Основная особенность предлагаемой реализации генетического алгоритма состоит в использовании для оценивания приспособленности популяции эксперимента на имитационной модели (рис. 1). Это обстоятельство предъявляет особые требования к выбранному способу

$$
-236-
$$




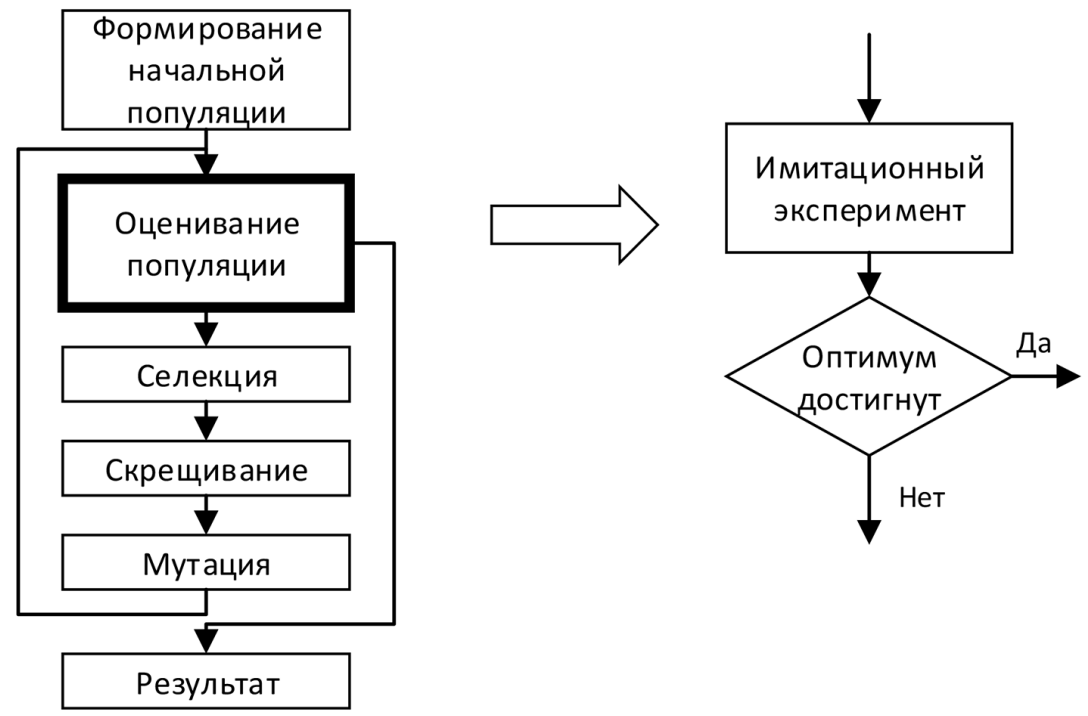

Рис. 1. Оптимизация на основе генетического алгоритма

Fig. 1. Genetic Algorithm Optimization

имитационного моделирования: он должен давать оценку функции приспособленности максимально быстро. Поэтому в некоторых случаях имитационную модель, очевидно, придется упрощать и обобщать. В рассмотренном ниже примере это не потребовалось.

Представленная схема связана с пошаговым конструированием вариантов с помощью моделирования, в процессе которого имитируется прохождение материальных потоков через производственный участок.

Генетический алгоритм выбран по следующим основным причинам.

1. Большая размерность задачи. Пространство поиска очень велико, и задействовано большое количество параметров.

2. Сравнительно высокая скорость и эффективность по сравнению с традиционными методами.

3. Наличие потенциальной возможности реализации параллельных вычислений.

4. Генетический алгоритм предоставляет список «хороших» решений, а не просто одно решение. Для рассматриваемой задачи это является важным свойством.

5. Всегда получает решение задачи, которое со временем становится лучше.

\section{3. Описание объекта исследования}

Рассматривается предприятие, относящееся к среднесерийному производству, выпускающее подъемно-транспортное оборудование. Организационная структура предприятия представлена обрабатывающими и сборочными участками, а также вспомогательным хозяйством. Рассматривается участок листовой металлообработки в обрабатывающем производстве. В цехе листовой металлообработки используется оборудование, перечисленное в табл. 1.

При составлении маршрута изготовления детали производится сокращенное описание всех технологических операций в маршрутной карте в последовательности их вы- 
Таблица 1. Технологическое оборудование участка

Table 1. Technological equipment of the site

\begin{tabular}{|l|l|}
\hline № & \multicolumn{1}{c|}{ Наименование оборудования } \\
\hline 1 & Гильотина ZDM \\
\hline 2 & Гильотина VIMERCATI \\
\hline 3 & Вертикально-гибочный гидравлический пресс VIMERCATI \\
\hline 4 & Вертикально-гибочный гидравлический пресс ZDM \\
\hline 5 & Координатно-пробивной пресс Euromac \\
\hline 6 & Координатно-пробивной пресс YANGLI \\
\hline
\end{tabular}

Таблица 2. Технологические маршруты

Table 2. Technological routes

\begin{tabular}{|l|c|c|c|c|c|c|c|c|c|c|c|c|c|c|c|c|c|c|}
\hline \multicolumn{1}{|c|}{ № маршрута } & 1 & 2 & 3 & 4 & 5 & 6 & 7 & 8 & 9 & 10 & 11 & 12 & 13 & 14 & 15 & 16 & 17 & 18 \\
\hline Набор станков & 1 & 2 & 1 & 1 & 2 & 2 & 1 & 1 & 2 & 2 & 2 & 2 & 1 & 1 & 2 & 2 & 2 & 2 \\
в маршруте & & & 3 & 4 & 3 & 4 & 5 & 5 & 5 & 5 & 6 & 6 & 5 & 5 & 5 & 5 & 6 & 6 \\
& & & & & & & 3 & 4 & 3 & 4 & 3 & 4 & 1 & 1 & 2 & 2 & 2 & 2 \\
& & & & & & & & & & & & & 3 & 4 & 3 & 4 & 3 & 4 \\
\hline
\end{tabular}

полнения без указания переходов и технологических режимов, но с указанием типа оборудования. Маршрутное описание процессов обычно является основным в единичном и мелкосерийном производствах и сопроводительным (дополнительным) в других типах производств.

В исследуемом металлообрабатывающем цехе могут быть реализованы следующие технологические маршруты (табл. 2).

В качестве программной среды моделирования выбран Tecnomatix Plant Simulation. Выбор программы обусловлен интеграцией средств имитационного моделирования и генетического алгоритма в одной среде и большим количеством средств анализа процессов [10, 11]. Формально имитационная модель в программной среде Тесnomatix реализована на основе библиотеки стандартных модельных элементов программы (рис. 2).

Параметризация имитационной модели. Параметризация модели осуществлена созданием набора таблиц, содержащих данные о заказах, операциях и маршрутах.

Таблица «Заказ_наряд» предназначена для постановки сменного задания. В таблице используются различные типы данных. В первом столбце «object» - графический объект (в данном случае деталь). Во втором столбце «integer»- целочисленное значение, представляющее количество деталей на запуск. В третьем столбце «string» - строковый тип данных, определяющий имя детали. В четвертом столбце «table» - таблица атрибутов.

Таблица «Справочник_операций» - предназначена для переопределения атрибутов в данном случае на станках при прохождении графического объекта (детали) (табл. 3). Восьмой столбец идентифицирует маршрут детали в моделируемой системе. Структура маршрута в привязке к конкретному оборудованию определена в таблице «Маршруты». 


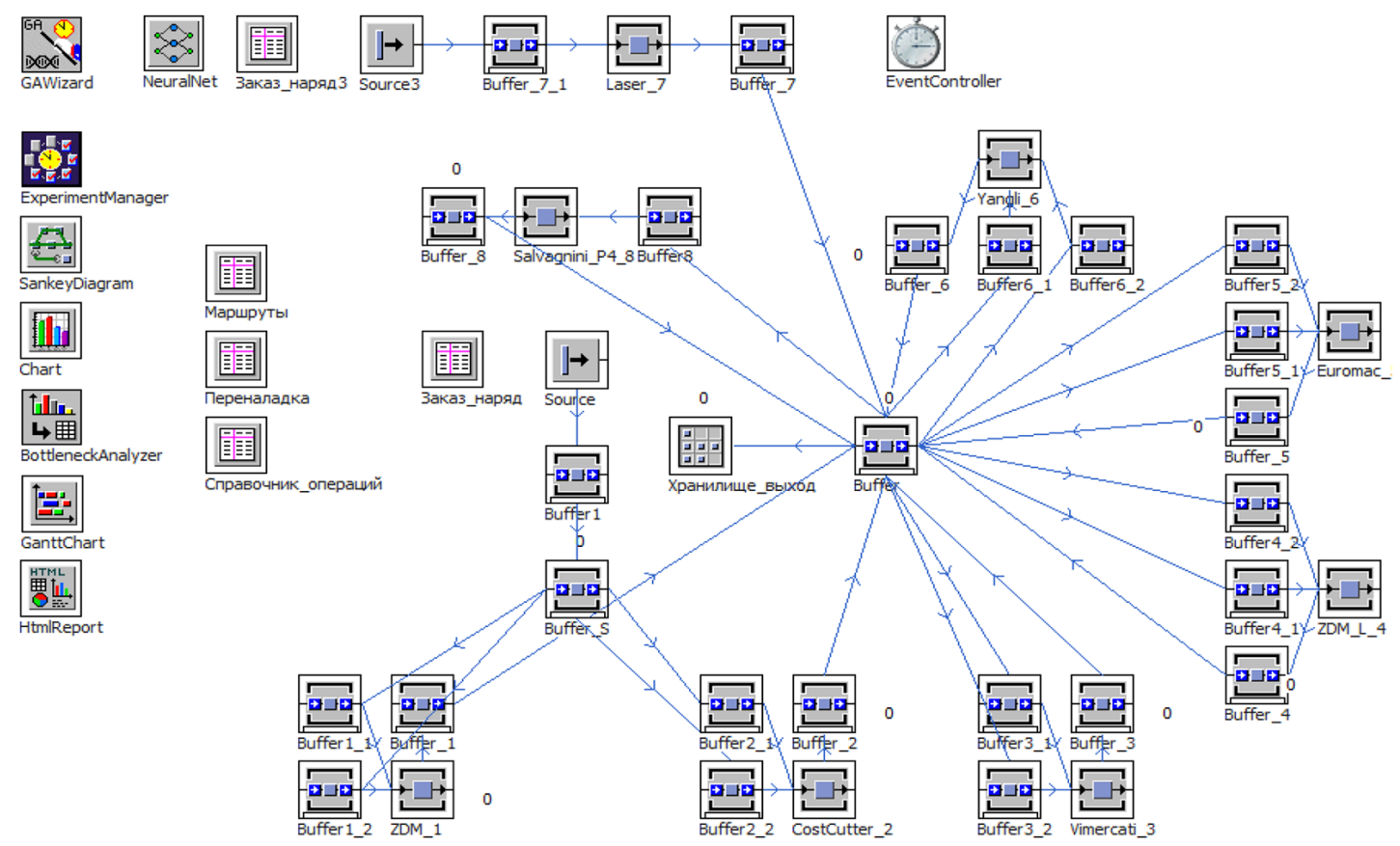

Рис. 2. Структура имитационной модели производственного процесса

Fig. 2. The structure of the simulation model of the production process

Таблица 3. Справочник операций

Table 3. Operations reference

\begin{tabular}{|c|c|c|c|c|c|c|c|c|}
\hline & {$\left[\begin{array}{l}\text { string } \\
1\end{array}\right.$} & $\begin{array}{l}\text { time } \\
2\end{array}$ & $\begin{array}{l}\text { time } \\
3\end{array}$ & $\begin{array}{l}\text { time } \\
4\end{array}$ & $\begin{array}{l}\text { time } \\
5\end{array}$ & $\begin{array}{l}\text { time } \\
6\end{array}$ & $\begin{array}{l}\text { time } \\
7\end{array}$ & $\begin{array}{l}\text { integer } \\
8\end{array}$ \\
\hline string & Name & Time_operation_1 & Time_operation_2 & Time_operation_3 & Time_operation_4 & Time_operation_5 & Time_operation_6 & M \\
\hline 1 & RRR & 20.0000 & 32.0000 & 15.0000 & 35.0000 & 18.0000 & 24.0000 & 17 \\
\hline 2 & RRR1 & 40.0000 & 23.0000 & 16.0000 & $1: 04.0000$ & 15.0000 & 32.0000 & 14 \\
\hline 3 & RRR2 & 35.0000 & 36.0000 & 45.0000 & 17.0000 & 13.0000 & 20.0000 & 16 \\
\hline 4 & RRR3 & 23.0000 & 13.0000 & 34.0000 & 23.0000 & 23.0000 & 45.0000 & 15 \\
\hline 5 & RRR4 & 43.0000 & 23.0000 & 32.0000 & 33.0000 & 47.0000 & 42.0000 & 10 \\
\hline
\end{tabular}

\section{4. Оптимальное планирование производства}

Рассмотрим начальный вариант работы системы. Сформировано некоторое случайное распределение чередования партий деталей в маршрутах. На рис. 3 приведена имитационная модель производственной системы, в которой моделируется процесс обработки партий десяти наименований деталей на четырех станках (ZDM, VIMERCATI № 1, Euromac, VIMERCATI № 2). В результате моделирования цикл обработки всех деталей составил 5 ч 16 мин. Анализ результатов показывает, что оборудование загружено неравномерно и имеется большой объем незавершенного производства в системе.

Для оптимизации производственных процессов Tecnomatix Plant Simulation имеет в своем составе модуль оптимизации на основе генетического алгоритма. Применение генетического алгоритма предполагает предварительный выбор его параметров. Для решения задается 


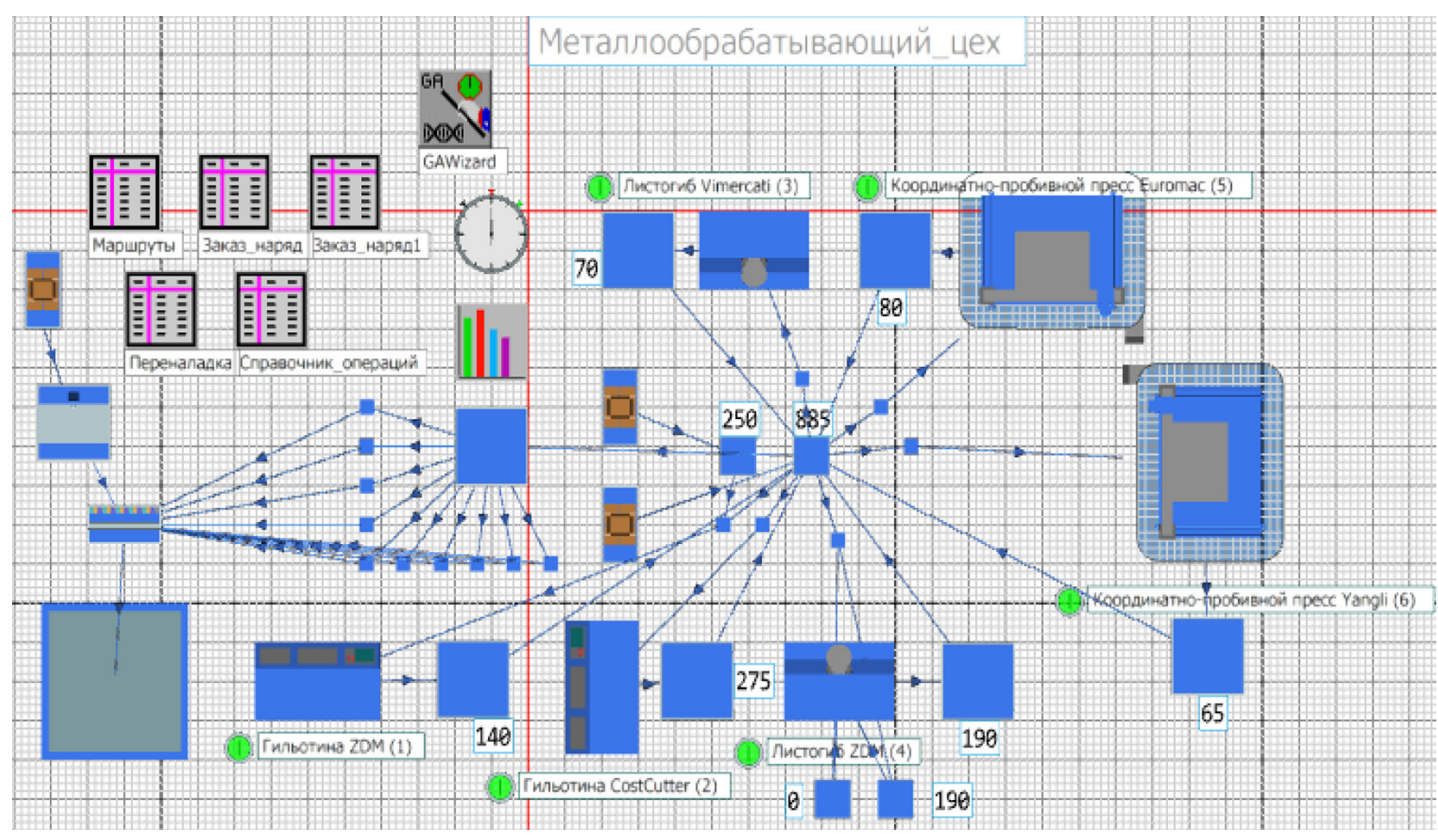

Рис. 3. Имитационный эксперимент с моделью

Fig. 3. Simulation experiment with a model

количество поколений, которое показывает количество решений задачи и размер поколения, который соответствует числу вариантов целевой функции в каждом решении. Исходя из требований к скорости и качеству решения, экспертным путем определили количество особей в популяции в каждом поколении (100 шт.).

Для ввода порядка следования деталей в модель создана еще одна таблица для параметризации. В качестве параметров оптимизации выбраны последовательности запуска заготовок каждого типа в производство (рис. 4). Модельным отображением выбранной целевой функции является общее время обработки всех деталей (переменная simtime).

Процесс поиска решения сопровождается изменением функций приспособленности. На рис. 5, 6 показаны значения функции приспособленности для первого и последнего поколений. Видно, что среднее значение целевой функции, представляющей общее время процесса в секундах, уменьшается. График на рис. 7, показывает, что минимальное значение практически устанавливается уже к 13 -му поколению и незначительно улучшается к 20 -му поколению.

В результате оптимизации получена новая последовательность запуска заготовок на обработку. Величина целевой функции после оптимизации для наилучшей хромосомы составила

\begin{tabular}{c|l|l|l|l}
\hline string & Parameter: & root.3ака3_наряд & Parameter: & root.3ака3_наряд1 \\
\cline { 2 - 5 } 1 & Последовательнос... & root.Заказ_наряд & Последовательнос... & root.3аказ_наряд1 \\
\hline 2 & 3 элементов & & 7 элементов & \\
\hline
\end{tabular}

Рис. 4. Настройка параметров оптимизации

Fig. 4. Setting optimization parameters 


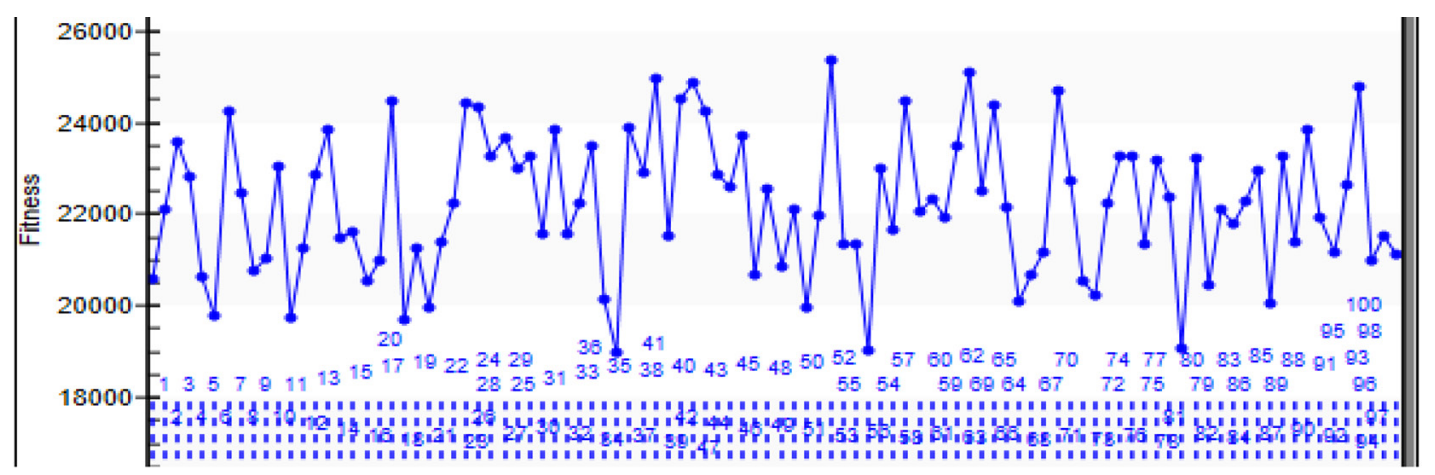

Рис. 5. Функции приспособленности 1-го поколения

Fig. 5. Fitness functions of the $1^{\text {st }}$ generation

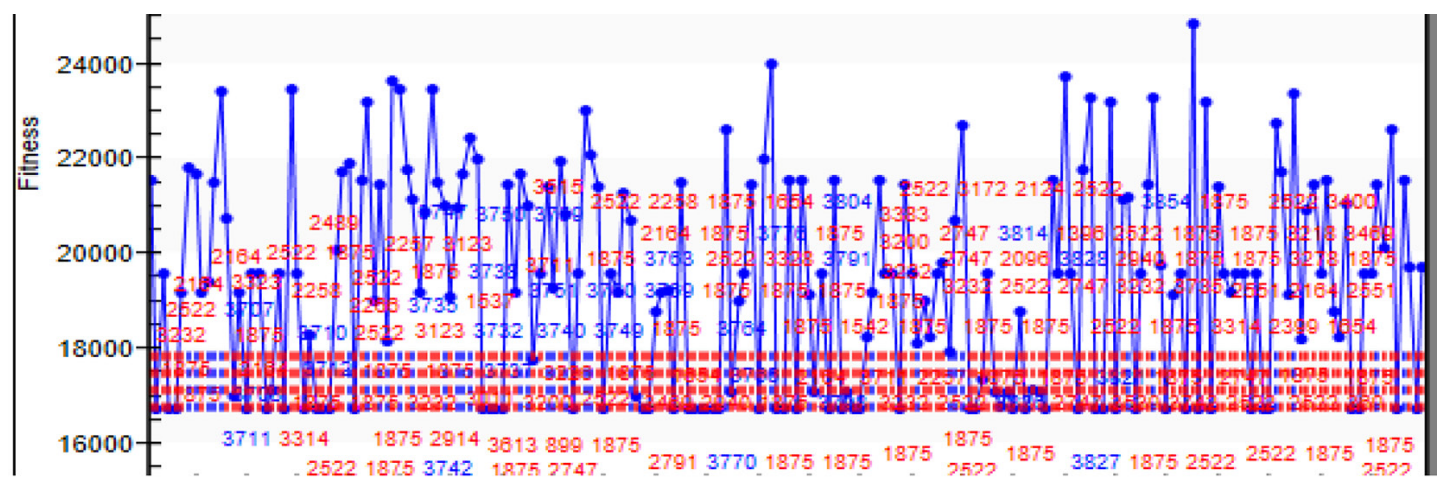

Рис. 6. Функции приспособленности 20-го поколения

Fig. 6. Fitness functions of the $20^{\text {th }}$ generation

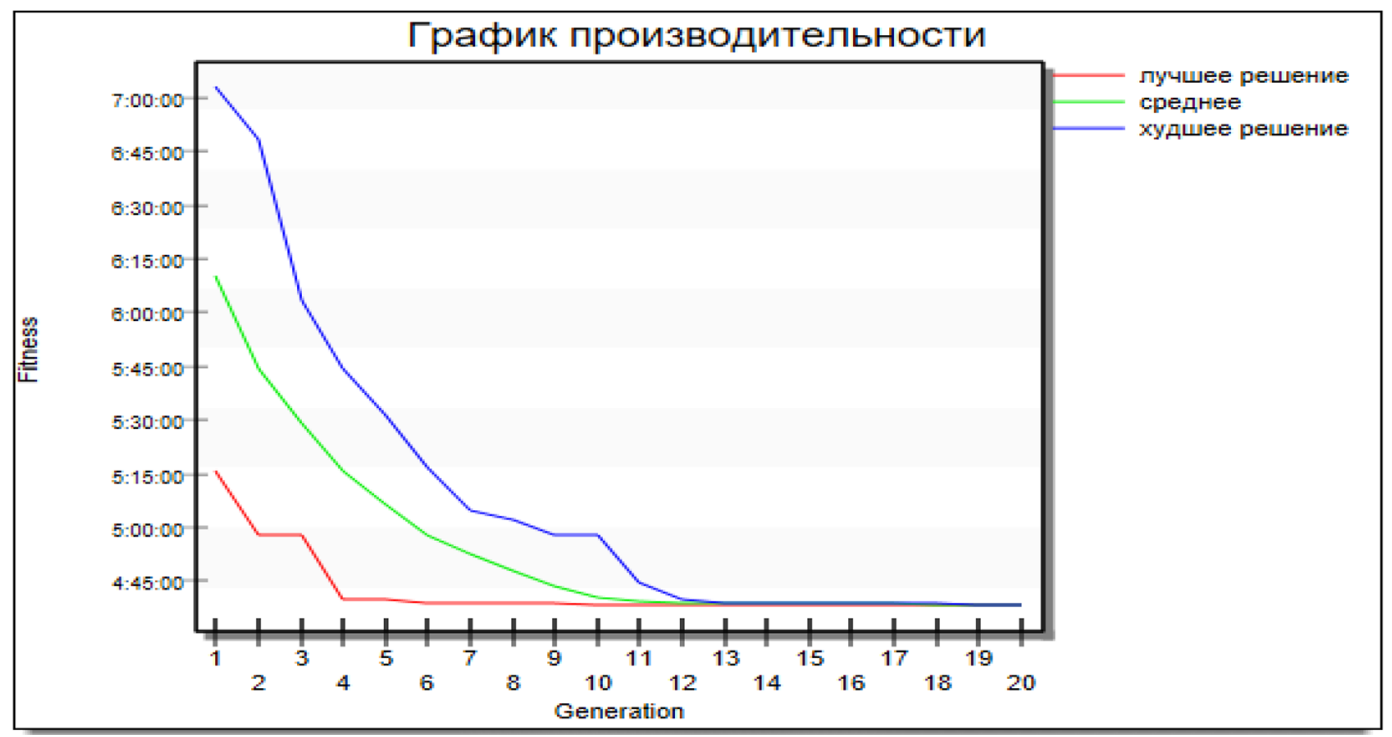

Рис. 7. Эволюционное изменение целевой функции по поколениям

Fig. 7. Evolutionary change in objective function by generation 
4 ч 37 мин. Таким образом, время обработки всех партий заготовок на станках уменьшилось на 29 мин. Сокращение произошло за счет перераспределения загрузки оборудования и сокращения общего времени переналадок.

\section{Заключение}

По итогам работы по тематике статьи получены следующие научные и научнопрактические результаты. В рамках оптимизационно-имитационного подхода предложен подход решения задачи предварительного планирования производства на основе предварительной параметризации имитационной модели генетическим алгоритмом. Описанный подход апробирован при решении задачи управления типичной дискретной производственной системой на основе специализированного и актуального программного обеспечения.

Основные преимущества и недостатки данного подхода соответствуют особенностям генетического алгоритма. Явным преимуществом выступает возможность использования различных целевых функций разного уровня сложности, возможность работы с большим количеством параметров, возможность настройки алгоритма. Используемые программные средства скрывают от пользователя некоторые параметры процедуры оптимизации. В то же время остается возможность остановки алгоритма на любом шаге решения при достижении результатов, устраивающих исследователя с точки зрения допустимых сроков выпуска продукции и других параметров. Для практических задач это позволяет получить если не оптимальное, то близкое к нему решение.

Предложенный подход может быть применен и для других видов и форм представления имитационных моделей при наличии возможности управления имитацией, например, для достаточно популярного инструмента моделирования - формализмов сетей Петри.

\section{Список литературы / References}

[1] Аничкин А.С. Объектно-ориентированная среда для разработки приложений теории расписаний, дисс. канд. физ.-мат. наук. М., 2018, 168 с. [Anichkin A. S. An object-oriented environment for developing scheduling theory applications, Diss. of the cand. of phys. and math. Sci. Moscow, 2018, 168 p. (in Russian)]

[2] Коваленко Ю.В. Сложность некоторых задач теории расписаний и эволюционные алгоритмы их решения, дисс. канд. физ.-мат. наук. Омск, 2013, 129 с. [Kovalenko Yu. V. The complexity of some problems in scheduling theory and evolutionary algorithms for solving them, Diss. of the cand. of phys. and math. Sci. Omsk, 2013, 129 p. (in Russian)]

[3] Сочнев А.Н. Сетевые модели в системах управления производством. Красноярск: Сиб. федерал. ун-т, 2014. 162 c. [Sochnev A. N. Network models in production management systems. Krasnoyarsk, Sib. Fed. Univ., 2014, 162 p. (in Russian)]

[4] Васильев А.С., Матвейкин В.Г. Модификация генетического алгоритма для решения задачи календарного планирования с ограниченными ресурсами, Вестник Тамбовского государственного технического университета, 2008, 14(2), 310-314 [Vasiliev A.S., Matveykin V.G. Modification of the genetic algorithm to solve scheduling problems with limited resources, J. of the Tambov St. Tech. Univ., 2008, 14(2), 310-314 (in Russian)]

[5] Катаев А.В., Катаева Т.М., Макарова Е.Л. Управление проектами: математические модели оптимального назначения исполнителей проектных работ, Известия Саратовского 
университета, 2016, 3, 294-299 [Kataev A. V., Kataeva T. M., Makarova E. L. Project management: mathematical models of the optimal assignment of design work performers, Izv. Saratov Univ., 2016, 3, 294-299 (in Russian)]

[6] Костюк Ю.Л. Эффективная реализация алгоритма решения задачи коммивояжера методом ветвей и границ, Прикладная дискретная математика, 2013, 2 (20), 78-90 [Kostyuk Yu. L. Effective implementation of the algorithm for solving the traveling salesman problem using the branch and bound method, Appl. Discr. Math., 2013, 2(20), 78-90 (in Russian)]

[7] Гладков Л.А., Курейчик В.В., Курейчик В. М. Генетические алгоритмы. М.: ФИЗМАТЛИТ, 2006. 320 с. [Gladkov L.A., Kureichik V.V., Kureichik V.M. Genetic Algorithms, Moscow, FIZMATLIT, 2006, 320 p. (in Russian)]

[8] Цвиркун А.Д., Акинфиев В.К., Филиппов В.А. Имитационное моделирование в задачах синтеза структуры сложных систем (оптимизационно-имитационный подход). М.: Наука, 1985. 175 с. [Zvirkun A. D., Akinfiev V.K., Filippov V. A. Simulation in problems of synthesis of the structure of complex systems (optimization-simulation approach), Moscow, Nauka, 1985, 175 p. (in Russian)]

[9] Ковалев .В., Ц Царев Р. Ю., Тюпкин М. В., Цветков Ю. Д. Оптимизационноимитационный подход к синтезу автоматизированных систем управления, Программные продукты и системы, 2007, 3, 73-74. [Kovalev I.V., Tsarev R. Yu., Tyupkin M.V., Tsvetkov Yu.D. Optimization and simulation approach to the synthesis of automated control systems, Soft. Prod. and Syst., 2007, 3, 73-74 (in Russian)]

[10]Чижов М.И., Скрипченко Ю.С., Гусев П.Ю. Автоматизация и оптимизация технологических процессов в Tecnomatix Plant Simulation, Вестник Воронежского государственного технического университета, 2011, 7(12-1), 36-38. [Chizhov M.I., Skripchenko Yu.S., Gusev P. Yu. Automation and optimization of technological processes at Tecnomatix Plant Simulation, J. of Voronezh St. Tech. Univ., 2011, 7(12-1), 36-38 (in Russian)]

[11] Чижов М.И., Скрипченко Ю.С., Гусев П.Ю. Моделирование технологических процессов в Tесnomatix Plant Simulation, Вестник Воронежского государственного технического университета, 2011, 7, 18-20 [Chizhov M.I., Skripchenko Yu.S., Gusev P. Yu. Process Modeling at Tecnomatix Plant Simulation, J. of Voronezh St. Tech. Univ., 2011, 7, 18-20 (in Russian)] 